\title{
Identifying Nurse Education Needs with Documentation Audits
}

\author{
Alexandra T. Bambrick ${ }^{1}$, Emmanuel Iraguha ${ }^{1}$ \\ ${ }^{1}$ University Teaching Hospital of Butare, Rwanda
}

\section{Background}

In July 2014, the Intensive Care Unit (ICU) at the University Teaching Hospital of Butare (CHUB) implemented a new daily nursing flow sheet. The use of a detailed flow sheet assessment had never been implemented as a care modality in the ICU at CHUB. In October 2014, a documentation audit tool was developed to identify areas of educational needs for staff nurses. The goal of this project was to increase nurses' knowledge and understanding of clinical care that would increase quality of care as evidenced by improved assessment and documentation compliance as the hospital prepares for accreditation.

\section{Description}

The CHUB ICU has six beds and a staff of 16 nurses. One flow sheet was utilized in a 24-hour period. Documentation was started in July 2014 and audits were conducted retrospectively on monthly basis starting from October 2014. The findings were presented to all staff members including physicians. The audits will continue till June 2015. Using hospital accreditation as a framework, focus areas for each month were identified and educational lectures and case studies were developed to increase the knowledge of the ICU staff. A collaborative effort between a Rwandan and an American nurse educator developed and implemented the lectures and performed the retrospective chart audits. The focus areas identified from the audits included pain assessment and management, advanced assessment, accountability, fluid intake and output, and interventions such repositioning. The staff members were rewarded for meeting the set goals for each month and were encouraged to attend lectures. Staff members who passed a final written examination will receive an educational certificate.

\section{Lessons Learned}

A retrospective analysis showed that the flow sheet documentation compliance was initially at $40 \%$ for both August and September 2014, with a significant increase to 73\% in February 2015, and a dip to 68\% in March 2015. The decrease in March has re-motivated the staff to continue to improve. Project limitations include that documentation audits only reflect what was written, and does not validate nursing actions. Cultural differences have led to the development and implementation of educational topics not reflected in the documentation audit such as professional and institutional accountability rules and regulations for nurses. Conclusions: As nurses in Rwanda implement the change in clinical practice following this study, detailed nursing assessment and documentation will become more important. The development of documentation audit tools helped identification of educational needs of staff members and facilitated the use of detailed documentation. Increasing nursing knowledge has become crucial for Rwandan nurses as they strive towards professional nursing practice.

Key words: accreditation, audit, compliance, documentation, flow sheet 